\title{
Allergy to antibiotics in children: Perception versus reality
}

\author{
JM Langley MD MSc, S Halperin MD
}

A ntibiotics are the most commonly prescribed drugs in children and are most likely to be associated with adverse reactions (1-3). Less than $25 \%$ of all adverse drug reactions are due to allergies to the drugs (4). Although these illnesses are usually not severe enough to lead to hospital admission (5), they are a concern for parents, and children who are antibiotic-allergic have an increased number of medical visits and antibiotic prescriptions, and higher antibiotic costs (6) than other children. Clinicians are often hesitant to prescribe an antibiotic to patients with suspected, but unproven, immunoglobulin E ( $\lg E$ )mediated allergies $(7,8)$ because of the potential risk of lifethreatening anaphylaxis. The consequence of avoiding firstline agents is that alternative antibiotics are usually more expensive, have a broader spectrum of antimicrobial coverage and, therefore, are more likely to alter normal flora, and to have more side effects (9). The purpose of this note is to review briefly antibiotic allergy in children and clinical approaches to children with suspected antibiotic allergies.

An adverse reaction to an antibiotic is "any response to a drug which is noxious and unintended, and which occurs at doses used in man for prophylaxis, diagnosis, or treatment" (10). Generally, hypersensitivity or allergic drug reactions are categorized according to the immunopathogenic mechanisms outlined by Gell and Coombs (11) (Table 1), and more than one mechanism can be operating simultaneously (4). The true prevalence of antibiotic allergy is unknown. In ambulatory children followed in prospective studies, the incidence of adverse drug reactions ranges from $0.75 \%$ to $4.5 \%(1-3,12,13)$. The percentage of those attributable to drug allergy is not known because few population-based studies are available, and most reports are of individual patients or groups of patients. Furthermore, the pathophysiological basis of adverse reactions to many antibiotics is not known, although it may be labelled as allergic in nature. For example, the serum sickness-like illness that occurs in about $0.06 \%$ of children receiving cefaclor (14) is likely due to a cytotoxic effect of the drug on cells (15), rather than immune complex deposition. Some commonly used antibiotics and the types of allergies associated with their use can be found in Table 2 .

Parental or patient reports of drug allergies always overestimate the true frequency. For example, when children with reported allergies to penicillin are subjected to skin testing, a range of $0 \%$ to $34 \%$ will have an IgE-type reaction (16-20). A small number of children have allergies to multiple antibiotics (21); the etiology of this phenomenon is not clear.

The variation in the reported frequency of allergies among those who are labelled as antibiotic-allergic before skin testing is likely due, in some part, to differences in physician management of the initial clinical event that leads to suspicion of drug allergy. Physician documentation of the clinical findings of the suspected allergic reaction

TABLE 1

\section{Classification of allergic drug reactions}

\begin{tabular}{|c|c|}
\hline Type I: & IgE-mediated (eg, anaphylaxis to penicillin) \\
\hline Type II: & cytotoxic antibodies (eg, penicillin-hemolytic anemia) \\
\hline Type III: & antigen-antibody complexes (eg, serum sickness) \\
\hline Type IV: & $\begin{array}{l}\text { cell-mediated immune reactions (eg, gramicidin/ } \\
\text { neomycin sulfate/polymyxin B sulfate [Neosporin }\end{array}$ \\
\hline
\end{tabular}

IgE Immunoglobulin $E$ 
TABLE 2

\section{Allergic reactions to some commonly used antibiotics in children}

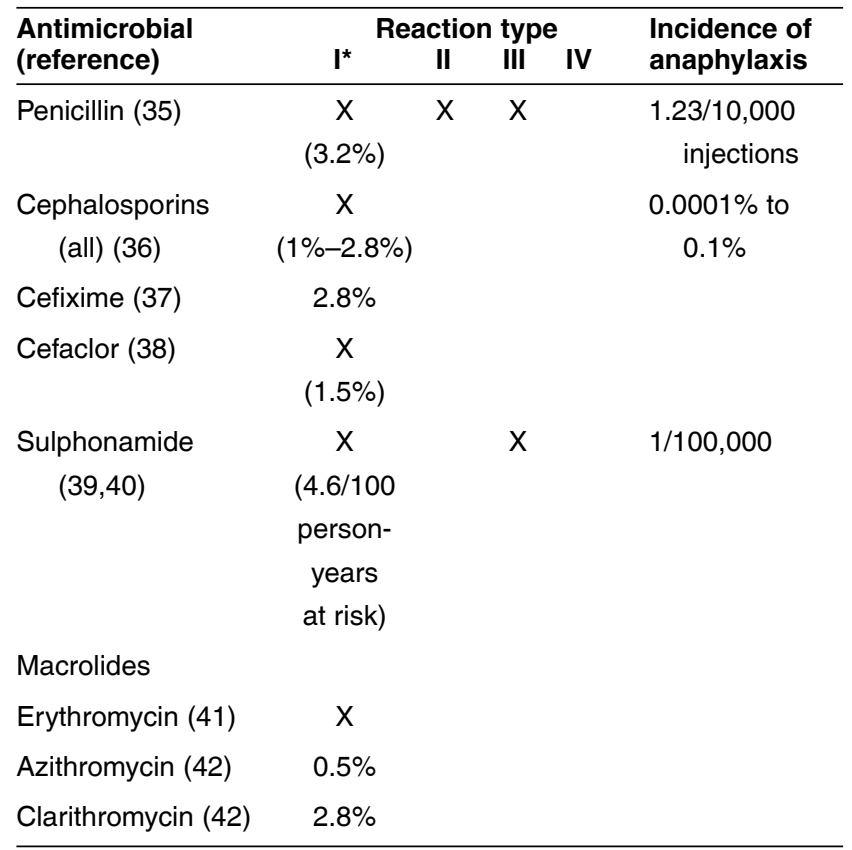

*Incidence is in brackets

may be incomplete (9), making interpretation difficult for subsequent care providers. Discrepancies between parental reporting of antibiotic allergy and confirmation in the health record (6) suggest either poor documentation or miscommunication as to the likely diagnosis when adverse drug reactions occur.

Clinical diagnosis of adverse reactions to antibiotics is difficult, and this may result in labelling the child as allergic for lack of a better explanation of the event. Antibiotics are prescribed frequently for febrile infectious illnesses in childhood, and many of these are associated with urticarial or other rashes. A morbilliform, nonurticarial rash may occur in up to $13 \%$ of patients who receive amoxicillin or ampicillin. These patients are not considered to be at risk for a life-threatening reaction to penicillin. The interaction between viral infections and certain antibiotics can result in adverse events that appear to be specific to certain viruses, for example, cutaneous reactions to ampicillin in acute infectious mononucleosis and to sulphonamides in patients with HIV infection (22). Reactions may be caused by excipients and additives in the antibiotic preparation, or by a drug previously taken by the patient (19). Although frequently a cause of physician and parent anxiety, the incidence of allergic cross-reactions to cephalosporins in patients who are allergic to penicillin is less than $2 \%$ (4).

The clinical evaluation of adverse drug reactions is difficult; therefore, systematic approaches have been proposed $(23,24)$ and, if implemented, will likely reduce the number of children being inappropriately labelled as allergic to antibiotics. Detailed algorithms have also been reported, but none has proven to be sufficiently sensitive and specific (24). An approach to the clinical evaluation of suspected adverse drug reactions is presented in Table 3 .

There is no single test or clinical finding that leads to a diagnosis of antibiotic allergy $(23,25-27)$. The only validated method for determining drug allergy in clinical practice is skin testing for IgE-mediated allergy to penicillin $(4,28)$. The radioallergosorbent test for serum IgE to penicillin is not recommended because of poor sensitivity and because it is only available for the major determinant of penicillin (4). There are no reliable skin tests routinely available for assessing allergic reactions to other antimicrobial agents (25). The development of immunological diagnosis of antibiotic allergy is limited because most drugs are incomplete antigens, or haptens (15). Haptens cannot stimulate hypersensitivity reactions until they bind to a carrier molecule. With the exception of penicillin, immunoreactive molecules have rarely been identified (28).

If the child's history is suggestive of an IgE-mediated allergy to penicillin, skin testing is an established method to determine whether a specific IgE response exists and to determine type I hypersensitivity $(4,28)$. The negative predictive value of a negative skin test is greater than $99 \%$ (29). Skin testing cannot detect non-IgE-mediated immunological reactions such as serum sickness and hemolytic anemia. The procedure consists of a scratch test on the volar surface of the forearm using preparations of the major and minor determinants of penicillin, and positive (histamine) and negative (saline) controls. If scratch testing is negative after $15 \mathrm{~min}$, intradermal testing is completed with the same agents. If no reaction is observed during the skin tests, an oral challenge with a standard dose of penicillin is given, and the child is observed for $1 \mathrm{~h}$. Generally, this clinic visit takes approximately $2.5 \mathrm{~h}$. If no adverse reaction is observed, then the family can be told that the risk of a life-threatening or serious reaction if the child takes penicillin is no greater than that of the general population. It is important that the parent and child are educated about the meaning of negative and positive test results, and that a system is in place to update the child's health record if penicillin allergy warning labels are to be removed from the chart $(16,30)$ and subsequent prescribing behaviour is to change.

Skin testing must be conducted by personnel who are familiar with the techniques in a setting capable of managing rare systemic reactions and with appropriate test materials $(4,28)$. Up to $90 \%$ of systemically administered penicillin is excreted in the urine, and a fraction is metabolized. The remaining fraction undergoes spontaneous degradation. The degradation products react with host proteins to form the penicilloyl group (major determinants) or form penicilloate, penicilloylamine and penilloate (minor determinants) (31). Because no commercially available formulation of a minor determinant mixture is available, various locally prepared reagents have been used, and only $40 \%$ of allergists surveyed in the United States use a minor determinant mix- 


\section{TABLE 3}

Approach to a suspected adverse drug reaction

1) Clinical diagnosis

Obtain a detailed history of the name of the drug(s), indications for the drug, concurrent infections and other clinical features, time lapse between when the drug was taken and the onset of the reaction, and history of resolution of symptoms with drug withdrawal

Perform physical examination for skin rashes (urticaria, angioedema, maculopapular rashes, dermatitis and vasculitis) and their distribution. Drug reactions may involve any system

2) Analysis of drug exposure

Review current and past exposures to this drug (name, formulation, concentration, dose and known excipients)

3) Differential diagnosis

Consider all possible clinical diagnoses, including the underlying condition. Is the reaction in keeping with known adverse reactions to the drug? If Stevens-Johnson syndrome, exfoliative dermatitis, hepatotoxicity or anaphylaxis occurred, refer the patient to a specialist for assessment

4) Literature review

Search medical literature for evidence showing that the drug in question is associated with the adverse event. If there is no association, reconsider the diagnosis and consider additives to the drug preparation

5) Confirmation

Complete skin testing for penicillin, refer to specialist

6) Report to drug regulatory authorities and the drug manufacturer

7) Advice to the patient

Notify the patient and other health care professionals involved in the patient's care (MedicAlert, MedicAlert Foundation, USA). Determine alternate antibiotic choices if necessary

Data from references $23,25,43$

ture when they perform skin testing (8). Although only $14 \%$ of true penicillin allergy is to a minor determinant, these responses have a higher likelihood of being anaphylactic reactions. Only minor determinant mixtures are recommended to detect allergy to minor determinants $(4,28)$. In Canada, a number of physician specialists (paediatric allergists and immunologists, pharmacologists, infectious disease specialists) $(20,21)$ provide skin testing for penicillin allergy and evaluation of patients with suspected drug hypersensitivity.

\section{RECOMMENDATIONS}

Use antibiotics wisely. The Canadian Paediatric Society recommends that antimicrobial therapy should be limited to situations in which there is a clear indication and should be administered for the shortest effective duration (32). The appropriate use of antimicrobial agents decreases the incidence of adverse drug reactions.

If a suspected allergic adverse drug reaction occurs in a child taking antimicrobial agents, perform a careful clinical assessment to determine whether the clinical event is an adverse drug reaction and whether it is potentially allergic in nature. The nature of the reaction should be carefully documented in the child's health record. Suspected adverse drug reactions should be reported to the Canadian Adverse Drug Reaction Monitoring Program of Health Canada. These reactions can be reported whether they are expected or not, regardless of their severity. Canadian Adverse Drug
Reaction reporting forms can be downloaded from the Therapeutic Products Directorate Web site (http://www.hcsc.gc.ca/hpb-dgps/therapeut/zfiles/english/guides/adr/ adr_guideline_e.html), copied from the Compendium of Pharmaceuticals and Specialties (33) or obtained from regional or national adverse drug reaction units.

If the adverse drug reaction is to penicillin and is thought to be a manifestation of an IgE-mediated allergy, arrange for skin testing. This may require referral to an adverse drug reaction clinic, an allergist or another specialist. Skin testing should only be conducted in settings in which personnel are familiar with the technique, use appropriate materials and are able to manage life-threatening drug reactions.

If the adverse drug reaction is to an antimicrobial agent other than a penicillin and is thought to be allergic in nature, avoid the use of the drug. If the patient is likely to need antibiotics in the future (eg, recurrent urinary tract infections), then the patient may benefit from referral to a specialist to determine which antimicrobial agent(s) is the most effective and safe choice.

If a child is allergic to an antibiotic and it is the only therapeutic choice, desensitization may be necessary. Desensitization protocols are available for several antibiotics, and describe administration of the drug in progressively increasing doses given by mouth (34) and intravenously (28). These protocols must be performed in a hospital setting by experienced personnel. 


\section{REFERENCES}

1. Kramer MS, Hutchinson TA, Flegel KM, Naimark L, Contardi R, Leduc DG. Adverse drug reactions in general pediatric outpatients. J Pediatr 1985;106:305-10.

2. Menniti-Ippolito G, Raschetti R, Da Cas R, Giaquinto C, Cantarutti L. Active monitoring of adverse drug reactions in children. Italian Paediatric Pharmacosurveillance Multicenter Group. Lancet 2000;355:1613-4.

3. Cirko-Begovic A, Vrhovac B, Bakran I. Intensive monitoring of adverse drug reactions in infants and preschool children. Eur J Clin Pharmacol 1989;36:63-5.

4. Joint Task Force on Practice Parameters. Disease Management of Drug Hypersensitivity: A Practice Parameter. Ann Allergy Asthma Immunol 1999;83.

5. Mitchell AA, Lacouture PG, Sheehan JE, Kauffman RE, Shapiro S. Adverse drug reactions in children leading to hospital admission. Pediatrics 1988;82:24-9.

6. Kraemer M, Caprye-Boos H, Berman H. Increased use of medical services and antibiotics by children who claim a prior penicillin sensitivity. West J Med 1987;146:697-700.

7. Solensky R, Earl HS, Gruchalla RS. Clinical approach to penicillinallergic patients: A survey. Ann Allergy Asthma Immunol 2000;84:329-33.

8. Wickern GM, Nish WA, Bitner AS, Freeman TM. Allergy to beta-lactams: A survey of current practices. J Allergy Clin Immunol 1994;94:725-31.

9. Preston SL, Briceland LL, Lesar TS. Accuracy of penicillin allergy reporting. Am J Hosp Pharm 1994;51:79-84.

10. Karch FE, Lasagna L. Adverse drug reactions. A critical review. JAMA 1975;234:1236-41.

11. Gell P, Coombs R. Classification of allergic reactions responsible for clinical hypersensitivity and disease. In: Gell P, Coombs R, Lachmann P, eds. Clinical Aspects of Immunology. Oxford: Blackwell, 1975:761-81

12. Sanz E, Boada J. Adverse drug reactions in paediatric outpatients. Int J Clin Pharmacol Res 1987;7:169-72.

13. Woods CG, Rylance ME, Cullen RE, Rylance GW. Adverse reactions to drugs in children. BMJ 1987;294:869-70.

14. Prober C. Cepahalosporins: An update. Pediatr Rev 1998;19:118-27.

15. Borish L. Immune mechanisms of drug allergy. Immunol Allergy Clin North Am 1998;18:717-29.

16. Langley J, Halperin S, Bortolussi R. History of penicillin allergy and referral for skin testing: Evaluation of a pediatric program. 39th Annual Meeting of the Infectious Diseases Society of America. San Francisco, California. October 25-28, 2001.

17. Pichichero ME, Pichichero DM. Diagnosis of penicillin, amoxicillin, and cephalosporin allergy: Reliability of examination assessed by skin testing and oral challenge. J Pediatr 1998;132:137-43.

18. Mendelson LM, Ressler C, Rosen JP, Selcow JE. Routine elective penicillin allergy skin testing in children and adolescents: Study of sensitization. J Allergy Clin Immunol 1984;73:76-81.

19. Huang SW, Borum PR. Study of skin rashes after antibiotic use in young children. Clin Pediatr (Phila) 1998;37:601-7.

20. Warrington R, Simons F, Ho H, Gorski B, Tse K. Diagnosis of penicillin allergy by skin testing: The Manitoba experience. CMAJ 1978;118:787-91.

21. Park J, Matsui D, Reider M. Multiple antibiotic sensitivity syndrome in children. Can J Clin Pharmacol 2000;7:38-41.

22. Levy M. Role of viral infections in the induction of adverse drug reactions. Drug Saf 1997;16:1-8.
23. Shear N. Adverse reactions to drug therapy. In: Koren G, Prober C, Gold R, eds. Antimicrobial Therapy in Infants and Children. New York: Marcel Dekker, 1988:793.

24. Roujeau JC, Stern RS. Severe adverse cutaneous reactions to drugs. N Engl J Med 1994;331:1272-85.

25. Reider M. In vivo and in vitro testing for adverse drug reactions. Pediatr Clin North Am 1997:44:93-111.

26. Boguniewicz M, Leung DY. Hypersensitivity reactions to antibiotics commonly used in children. Pediatr Infect Dis J 1995;14:221-31.

27. Sullivan TJ. Pathogenesis and management of allergic reactions to penicillin and other beta-lactam antibiotics. Pediatr Infect Dis 1982;1:344-50

28. Bernstein IL, Storms WW. Practice parameters for allergy diagnostic testing. Joint Task Force on Practice Parameters for the Diagnosis and Treatment of Asthma. The American Academy of Allergy, Asthma and Immunology and the American College of Allergy, Asthma and Immunology. Ann Allergy Asthma Immunol 1995; 75:543-625.

29. Sogn DD, Evans R 3rd, Shepherd GM, et al. Results of the National Institute of Allergy and Infectious Diseases Collaborative Clinical Trial to test the predictive value of skin testing with major and minor penicillin derivatives in hospitalized adults. Arch Intern Med 1992;152:1025-32.

30. Harris AD, Sauberman L, Kabbash L, Greineder DK, Samore MH. Penicillin skin testing: A way to optimize antibiotic utilization. Am J Med 1999;107:166-8.

31. Solensky R, Mendelson LM. Systemic reactions to antibiotics. Immunol Allergy Clin North Am 2001;21:679-97.

32. Canadian Pediatric Society Infectious Diseases and Immunization Committee. Antimicrobial resistance: Implications for therapy of infections with common childhood pathogens. Paediatr Child Health 1996;1:51-5.

33. Compendium of Pharmaceuticals and Specialties, 37th edn. Ottawa: Canadian Pharmacists Association, 2002

34. Sullivan T, Yecies L, Shatz G, Parker C, Wedner H. Desensitization of patients allergic to penicillin using orally administered beta-lactam antibiotics. J Allergy Clin Immunol 1982;69:275-82.

35. Markowitz M, Lue HC. Allergic reactions in rheumatic fever patients on long-term benzathine penicillin G: The role of skin testing for penicillin allergy. Pediatrics 1996;97:981-3.

36. Kelkar PS, Li JT. Cephalosporin allergy. N Engl J Med 2001;345:804-9.

37. Tally F, Desjardins R, McCarthy E, Cartright K. Safety profile of cefixime. Pediatr Infect Dis J 1987;6:976-80.

38. Kammer R, Short L. Cefaclor: Summary of clinical experience. Postgrad Med J 1979;55(Suppl 4):93-7.

39. Uhari M, Nuutinen $M$, Turtinen J. Adverse reactions in children during long-term antimicrobial therapy. Pediatr Infect Dis J 1996;15:404-8.

40. Gutman L. The use of trimethoprim-sulfamethoxasole in children: A review of adverse reactions and indications. Pediatr Infect Dis J 1984:3:349-57.

41. Washington JI, Wilson W. Erythromycin: A microbial and clinical perspective after 30 years of clinical use (second of two parts). Mayo Clin Proc 1985;60:271-8.

42. Reed MD, Blumber JL. Azithromycin: A critical review of the first azilide antibiotic and its role in pediatric practice. Pediatr Infect Dis J 1997;16:1069-83.

43. Lavi S, Gold M. Drug Allergy: A practical approach. Pract Allergy Immunol 1990;5:4-8.

\section{CANADIAN PAEDIATRIC SOCIETY INFECTIOUS DISEASES AND IMMUNIZATION COMMITTEE}

Members: Drs Upton Allen, The Hospital for Sick Children, Toronto, Ontario; H Dele Davies, Division of Infectious Diseases, Alberta Children's Hospital, Calgary, Alberta; Joanne Embree, The University of Manitoba, Winnipeg, Manitoba, (chair); Joanne Langley, Department of Pediatrics, IWK Health Centre, Halifax, Nova Scotia; Mireille Lemay, Department of Infectious Diseases, Sainte-Justine Hospital, Montreal, Quebec; Gary Pekeles, The Montreal Children's Hospital, Montreal, Quebec (director responsible)

Consultants: Drs Noni MacDonald, Faculty of Medicine, Dalhousie University, Halifax, Nova Scotia; Victor Marchessault, Cumberland, Ontario Liaisons: Drs Scott Halperin, Department of Pediatrics, IWK Health Centre, Halifax, Nova Scotia (IMPACT); Susan King, Division of Infectious Diseases, The Hospital for Sick Children, Toronto, Ontario (Canadian Paediatric AIDS Research Group); Monique Landry, Direction de la santé publique de Laval, Laval, Quebec (Public Health); Larry Pickering, Centre for Pediatric Research, Norfolk, Virginia, USA (American Academy of Pediatrics, Committee on Infectious Diseases)

Principal authors: Drs Joanne Langley, Halifax, Nova Scotia; Scott Halperin, Halifax, Nova Scotia

The recommendations in this note do not indicate an exclusive course of treatment or procedure to be followed. Variations, taking into account individual circumstances, may be appropriate. This article also appears in Paediatr Child Health 2002;7(4):233-237. 


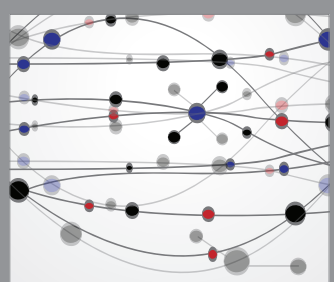

The Scientific World Journal
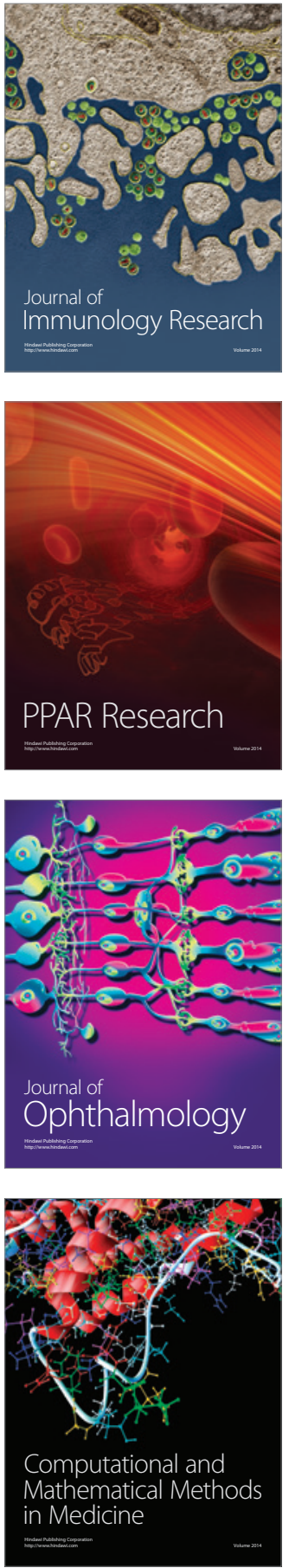

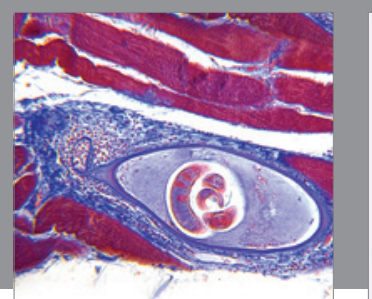

Gastroenterology Research and Practice

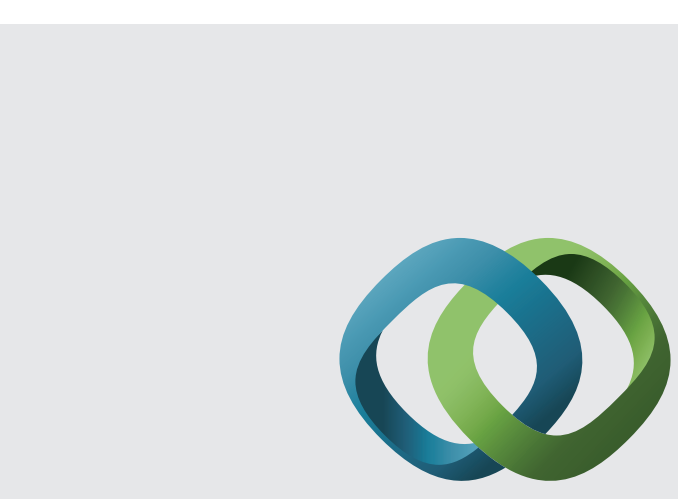

\section{Hindawi}

Submit your manuscripts at

http://www.hindawi.com
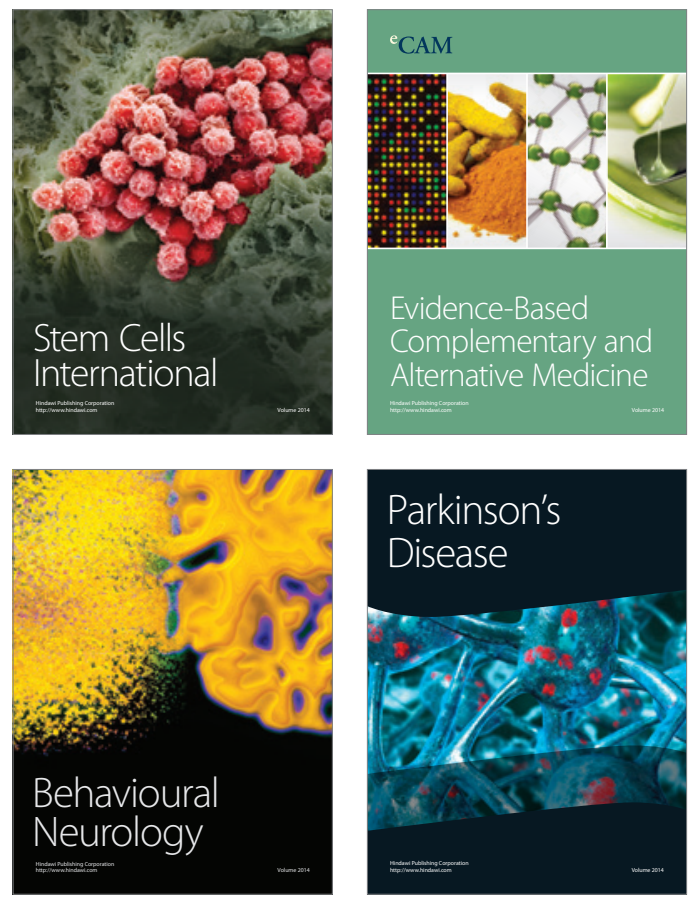
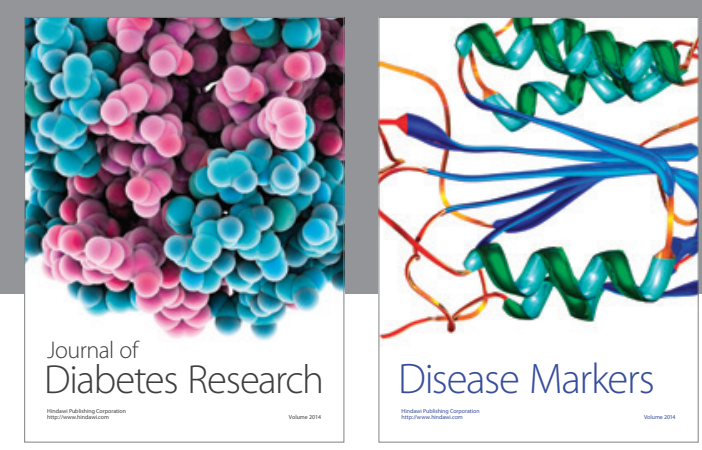

Disease Markers
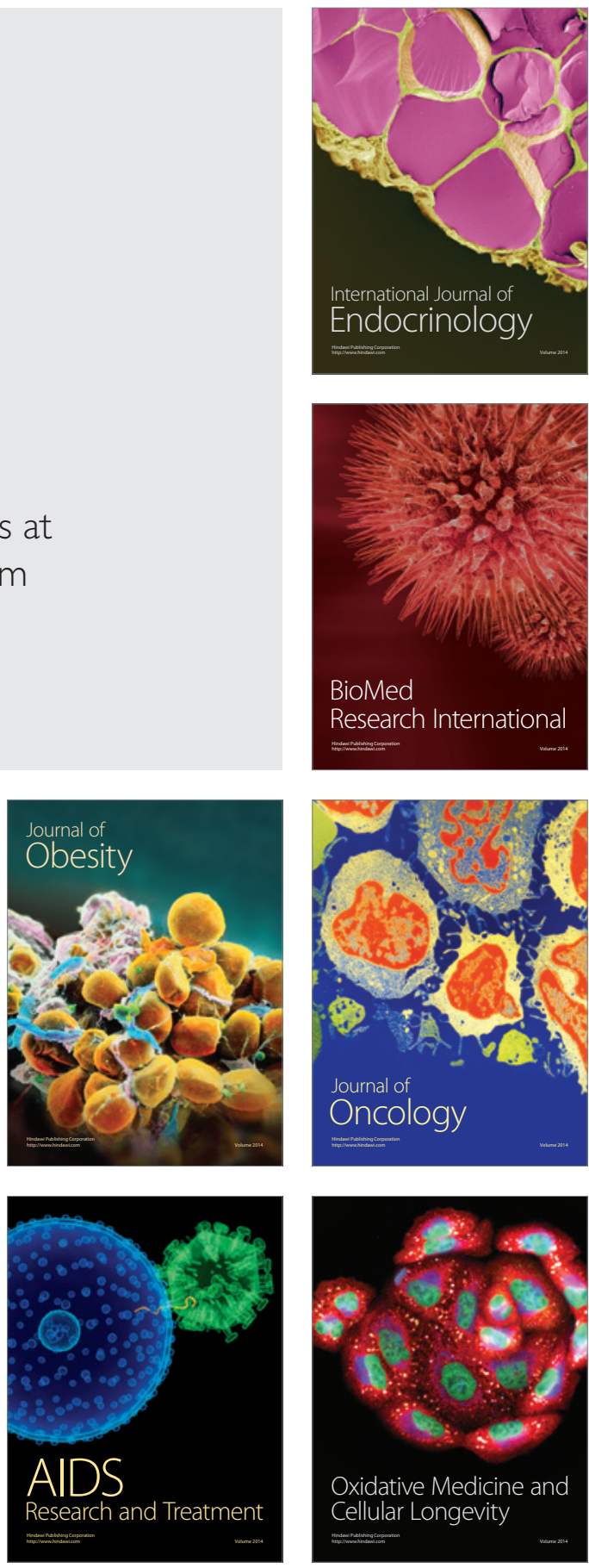